\title{
Towards development of unsteady near-wall interface boundary conditions for turbulence modeling
}

\author{
S.V. Utyuzhnikov* \\ School of Mechanical, Aerospace E' Civil Engineering, The University of Manchester, Manchester, M13 9PL, UK \\ Moscow Institute of Physics \& Technology, Dolgoprudny 141700, Russia
}

\section{A R T I C L E I N F O}

\section{Article history:}

Received 10 October 2012

Received in revised form

10 July 2014

Accepted 14 July 2014

Available online 18 July 2014

\section{Keywords:}

Domain decomposition

Interface boundary condition

Wall functions

Turbulence

Low-Reynolds-number model

Unsteady problems

URANS

Large eddy simulation

\begin{abstract}
A B S T R A C T
In near-wall turbulence modeling it is required to resolve a thin layer nearby the solid boundary, which is characterized by high gradients of the solution. An accurate enough resolution of such a layer can take most computational time. The situation even becomes worse for unsteady problems. To avoid timeconsuming computations, a new approach is developed, which is based on a non-overlapping domain decomposition. The boundary condition of Robin type at the interface boundary is achieved via transfer of the boundary condition from the wall. For the first time interface boundary conditions of Robin type are derived for a model nonstationary equation which simulates the key terms of the unsteady boundary layer equations. In the case of stationary solutions the approach is automatically reduced to the technique earlier developed for the steady problems. The considered test cases demonstrate that unsteady effects can be significant for near-wall domain decomposition. In particular, they can be important in the case of the wall-function-based approach.
\end{abstract}

(C) 2014 Elsevier B.V. All rights reserved.

\section{Introduction}

In near-wall turbulence modeling, an accurate enough resolution of the flow structure nearby the wall requires most computational time. This is due to the fact that the turbulent boundary layer has a very thin laminar sublayer nearby the wall caused by both the no-slip effect for the velocity and wall-blocking effect. Away from the wall the flow rapidly becomes turbulent that is described by high gradients in the solution. Even for stationary problems the resolution of the laminar sublayer takes up to $90 \%$ of computational time [1] because of an unavoidable mesh refinement. In industrial applications, it might be prohibitively expensive to use high resolution turbulence models especially in design where optimization is achieved via the solution of multiple direct problems.

In RANS modeling, the near-wall resolution is often significantly simplified via the replacement of the laminar sublayer by special Dirichlet boundary conditions called the wall functions. The wallfunction-based approach can be interpreted as a domain decomposition in which the near-wall region is either skipped or limited

\footnotetext{
* Correspondence to: School of Mechanical, Aerospace \& Civil Engineering, The University of Manchester, Manchester, M13 9PL, UK. Tel.: +44 1613063707.

E-mail address: s.utyuzhnikov@manchester.ac.uk.
}

by the nearest to the wall node. Because sublayer significantly determines the flow structure, this approach inevitably damages the accuracy. However, it might be efficient for some classes of problems due to a significant reduction of computational time. In the original form, the wall functions were based on the thin-plate analytical solution [2]. The wall-function-based approach is still very popular within industrial community because of its efficiency and reasonable accuracy. Yet, in most cases, the wall functions are semi-empirical and have some free parameters to be selected for special classes of problems. Their choice for new problems does not give much confidence in the obtained results unless additional calculations are carried out. The solution can also appear essentially mesh dependent, for example, if the first node is situated inside the viscous sublayer. Nowadays, there have been developed more universal wall functions which are determined by the solution in the core flow such as the scalable wall functions [3], adaptive wall functions [4], numerical and analytical wall functions [5]. Despite their advantages, all these boundary conditions are locally onedimensional. Therefore, their application to complex geometries is always problematic. For example in the case of a concave corner, there is formally the conflict of two normals at some near-wall nodes. In fact, this means the boundary condition must be nonlocal [6]. In addition, all wall functions have been derived only for steady RANS equations.

Nowadays, unsteady turbulent flows are usually simulated via either unsteady RANS equations (URANS) or large eddy simulation 
(LES). As the power of modern computers remarkably grows, the LES approach becomes more and more popular even in application to industrial problems. However, the application of LES to nearwall turbulent flows is very limited because the size of vortexes to be resolved vanishes to the wall. This requires the generation of a fine mesh and extremely increases computational time. As estimated in [7], the cost of a wall-resolved LES is proportional to $R e^{2.4}$, where $R e$ is the typical Reynolds number of the problem. To overcome this problem, hybrid RANS-LES models are often used [8-10]. The drawback of these approaches is the necessity of coupling two different governing equations.

One can distinguish weak and strong RANS-LES coupling [11], [12]. In the weak coupling, a single set of governing equations is derived to be applicable over entire computational region. In this strategy, RANS and LES models are blended in one way or another. This results in so-called global RANS-LES approaches. As indicated in [11], the global models lead to gray-areas which correspond to the region of blending. In such areas, the model is neither RANS nor LES. Conversely, this problem does not exist in the strong coupling with the use of RANS and LES in different regions (zones). In turn, the key problem of the zonal approach is related to RANS-LES interface. In the zonal strategy, the RANS models are usually represented by simplified wall models [13]. The wall model should provide to LES a set of approximate boundary conditions [14]. Physically, this means the wall model bring information about wall stresses to the interface boundary.

Similar to RANS, the wall-function based approach, first used [15], can be interpreted as a simplified zonal approach. There have been several attempts to modify the wall-function approach (see, e.g., [11,16]). However, as indicated in [17], the wall-function strategy can fail in application to essentially unsteady flows. Thus, the use of wall functions with LES and URANS is still questionable. It seems a more promising strategy is based on the use of the turbulent boundary-layer equations $[18,14,12,19]$. In this approach, approximate interface boundary conditions (IBC) are specified at some height above the physical location of the wall. These boundary conditions are of Dirichlet type and obtained via solution of thin-boundary-layer equations at each time step. As noted in [14], the implementation of such boundary conditions is not straightforward and can cause some non-physical effects such as the log-layer mismatch [12]. Therefore, this issue requires a careful elaboration.

In paper [20], the algorithm of transferring the boundary condition from the wall to an interface boundary was suggested. In particular, the near-wall interface boundary can correspond to the first node. In the case of RANS, the transfer of the boundary conditions requires an approximation of the turbulent viscosity coefficient [21]. It appears that for the locally one-dimensional transfer, the obtained boundary conditions are of Robin type. Therefore, in paper [22] they are called the Robin-type wall functions whereas they represent IBC. It is worth noting here that the conventional wall-function approach can be interpreted as an attempt to replace the physical no-slip boundary condition (in application to the velocity) by an approximate Dirichlet boundary condition. With this respect the title of Robin-type wall functions used in [22] for the IBCs can sound misleading.

The IBCs proved to be mesh independent and do not contain free parameters. The test cases, which were considered in [21,22], demonstrated that the solution is not very sensitive to the position of the interface boundary while the achieved accuracy being reasonably good. In [22], it was shown via the theory of Calderón-Ryaben'kii's potentials [23] that the IBCs [21] can be extended to the multi-dimensional case and represented by nonlocal boundary conditions. This approach was realized in [6] for a twodimensional model equation.
The technique of the boundary condition transfer cannot be immediately applied to the unsteady problem because of nonstationary terms. Therefore, this technique should be extended to unsteady problems and elaborated before its practical application. In the current paper, for the first time the technique of the boundary condition transfer [20] is developed and applied for unsteady problems. It is realized for an unsteady one-dimensional model equation, which simulates key features of the boundarylayer-type equations. The considered test cases demonstrate that the contribution of nonstationary terms cannot be neglected. In Section 2, the model equation suggested in paper [20] is extended to an unsteady formulation. The technique of non-overlapping domain decomposition is developed for the model equation in Section 3. On the basis of this approach, IBCs are derived in Section 4. Then, they are applied for some test cases in Section 5. In this Section, it is demonstrated that the unsteady effects can be significant for the accuracy of the approach. This result explains poor performance of the wall-function approach for unsteady flows obtained in [17]. As indicated in the Conclusion, the obtained result can be important for turbulence modeling in the case of essentially unsteady flows.

\section{Model equation}

Consider the following one-dimensional model equation:

$U_{t}=L_{y} U+R_{h}$,

defined on an interval $\Omega:=\left[0, y_{e}\right]$ with a Dirichlet boundary condition on the right-hand side:

$l_{y} U(0)=l_{0}$,

$U\left(y_{e}\right)=l_{1}$.

Here, $L_{y}$ is a linear differential operator of second order, which is supposed to be negative definite in the case of homogeneous boundary conditions; $l_{y}$ is a linear differential operator of first order. In particular, $l_{y}$ is equivalent to the unit for a Dirichlet boundary condition and derivative $\frac{d}{d y}$ for a Neumann boundary condition.

Eq. (1) represents the general form of the boundary-layer-type equation. The right-hand side $R_{h}$ is an appropriate source term which can simulate, e.g., the pressure gradient and any unsteady driving force in the momentum equation. Overall, Eq. (1) can be interpreted as a nonstationary generalization of the model equation suggested in [20].

In further consideration we assume that

$L_{y} u \equiv v u_{y}+\left(\mu u_{y}\right)_{y}$,

$R_{h}=c_{0}+f$,

where $v(y) \equiv c_{1} y^{\beta}, c_{1}, \beta>0$ are positive constants, $c_{0}$ is a constant, $f$ is an unsteady driving force, $\mu=\mu(y)$ is a function which simulates the effective viscosity coefficient:

$\mu=\left(1-\exp (-y / \epsilon)+\epsilon_{0}\right) / \operatorname{Re}$,

$R e, \epsilon, \epsilon_{0}$ are constant such that $\operatorname{Re} \gg 1, \epsilon \ll 1, \epsilon_{0} \ll 1$.

Consider on interval $(0, \delta): 0<\delta<y_{e}$ the space of functions that are supposed to be smooth enough and satisfy the homogeneous boundary conditions. Then, operator (3) proves to be negative definite. Indeed:

$\left(L_{y} u, u\right)=-\frac{1}{2}\left(u^{2}, v^{\prime}\right)-\left(\mu u_{y}, u_{y}\right) \leq 0$.

Here, $(\cdot, \cdot)$ is an inner product: $(a, b)=\int_{0}^{\delta} a b d y$.

On the other hand, Eq. (1) can be rewritten as follows:

$\rho^{\prime} U_{t}=L_{y}^{\prime} U+\rho^{\prime} R_{h}$ 
where

$L_{y}^{\prime} u \equiv\left(\mu \rho^{\prime} u_{y}\right)_{y}$,

and

$\rho^{\prime}=\exp \left(\int_{0}^{y} \frac{v}{\mu} d y^{\prime}\right)$.

One can prove, see e.g. [24], that the operator $L_{y}^{\prime}$ has the full system of eigenfunctions which are orthogonal with the weight of $\rho^{\prime}$.

\section{Non-overlapping domain decomposition}

Along with the domain $\Omega$, let us introduce domain $\Omega^{-}: \Omega^{-}:=$ $[0, \delta], 0<\delta<y_{e}$. Then, the domain $\Omega$ is split into two subdomains $\Omega^{-}$and $\Omega^{+}$, where $\Omega^{+}:=\left[\delta, y_{e}\right]$.

To realize a nonoverlapping domain decomposition, we introduce two auxiliary boundary value problems (BVPs) formulated in the domain $\Omega^{-}$. They are represented by

BVP 1:

$L_{y} V_{0}+c_{0}=0$

$l_{y} V_{0}(0)=0$

$V_{0}(\delta)=0$

and BVP 2:

$L_{y} V=0$,

$l_{y} V(0)=0$,

$V(\delta)=1$.

In addition, we consider two auxiliary initial BVPs (IBVPs), which are formulated as follows.

IBVP 1:

$W_{0 \mid t}=L_{y} W_{0}$,

$l_{y} W_{0}(0, t)=0$,

$W_{0}(\delta, t)=0$,

$W_{0}(y, 0)=w_{0}(y)$

where $w_{0}(y)=U(y, 0)-V_{0}(y)-V(y) U(\delta, 0)$, and IBVP 2:

$W_{t}=L_{y} W-\frac{d U_{\delta}}{d t} V$

$l_{y} W(0, t)=0$,

$W(\delta, t)=0$,

$W(0, y)=0$.

Then, the solution in the inner domain $\Omega^{-}$is represented by

$U=V_{0}+V U_{\delta}+W+W_{0}$,

where $U_{\delta}=U(\delta, t)$.

In the domain $\Omega^{-}$, there is the system of eigenfunctions $\Psi_{p}$ of the operator $L_{y}^{\prime}$ :

$L_{y}^{\prime} \Psi_{p}=-\lambda_{p} \rho^{\prime} \Psi_{p}, \quad p=1,2, \ldots$,

which is full and orthogonal: $\left(\Psi_{m}, \Psi_{n}\right)_{\rho^{\prime}} \equiv \int_{0}^{\delta} \rho^{\prime} \Psi_{m} \Psi_{n} d y=\delta_{m n}$, where $m, n=1, \ldots ; \delta_{m n}$ is the Kronecker symbol equal to 1 if $m=n$ and 0 , otherwise; $\lambda_{p}$, a $p$-th eigenvalue, which is real and positive.

Then, the solution to IBVP (7) is given by

$W_{0}=\sum_{1}^{\infty} C_{k}(t) \Psi_{k}$

Here, see e.g. [24]:

$C_{p}=a_{p} \exp \left(-\lambda_{k} t\right), \quad p=1,2, \ldots$ and

$a_{p}=\left(W_{0}, \Psi_{p}\right)_{\rho^{\prime}} \equiv \int_{0}^{\delta} \rho^{\prime} W_{0} \Psi_{p} d y, \quad p=1,2, \ldots$

Next, the solution to IBVP $(8)$ reads

$W=\sum_{1}^{\infty} \widehat{C}_{p} \Psi_{p}$,

where

$$
\begin{aligned}
\widehat{C}_{p}= & -\left(V, \Psi_{p}\right)_{\rho^{\prime}} \int_{0}^{t} \exp \left(-\lambda_{p}(t-\tau)\right) \frac{d U_{\delta}}{d \tau}(\tau) d \tau \\
= & -\left(V, \Psi_{p}\right)_{\rho^{\prime}}\left[U_{\delta}-U_{\delta}(0) \exp \left(-\lambda_{p} t\right)\right. \\
& \left.-\lambda_{p} \exp \left(-\lambda_{p} t\right) \int_{0}^{t} \exp \left(\lambda_{p} \tau\right) U_{\delta}(\tau) d \tau\right], \quad p=1,2, \ldots
\end{aligned}
$$

Then,

$W=-U_{\delta} \bar{W}+\sum_{1}^{\infty} \Phi_{p} \Psi_{p}$,

where

$\bar{W}=\sum_{1}^{\infty} \bar{C}_{k} \Psi_{k}$

$$
\begin{aligned}
\Phi_{p}= & \left(V, \Psi_{p}\right)_{\rho^{\prime}}\left(U_{\delta}(0) \exp \left(-\lambda_{p} t\right)\right. \\
& \left.+\lambda_{p} \exp \left(-\lambda_{p} t\right) \int_{0}^{t} \exp \left(\lambda_{p} \tau\right) U_{\delta}(\tau) d \tau\right), \quad p=1,2, \ldots
\end{aligned}
$$

and

$\bar{C}_{p}=\left(V, \Psi_{p}\right)_{\rho^{\prime}}$.

Thus, the entire solution in the inner domain is given by

$U=V_{0}+U_{\delta} V+\sum_{1}^{\infty} C_{k} \Psi_{k}-U_{\delta} \sum_{1}^{\infty} \bar{C}_{k} \Psi_{k}+\sum_{1}^{\infty} \Phi_{k} \Psi_{k}$

or

$U=V_{0}+U_{\delta} \bar{V}+\sum_{1}^{\infty}\left(C_{k}+\Phi_{k}\right) \Psi_{k}$

where

$\bar{V}=V-\sum_{1}^{\infty} \bar{C}_{k} \Psi_{k}$

This solution cannot be obtained until $U_{\delta}$ is known. However, as shown in the next Section, one can find the IBC without the solution in the outer domain $\Omega^{+}$.

\section{Unsteady interface boundary condition}

Having differentiated Eq. (9) at $y=\delta$, we arrive at the IBC, which is represented by a Robin boundary condition:

$U^{\prime}=V_{0}^{\prime}+\bar{V}^{\prime} U+\sum_{1}^{\infty}\left(C_{k}+\Phi_{k}\right) \Psi_{k}^{\prime}$.

Here and further, the prime denotes differentiation. This equation represents a boundary condition for function $U$ to be used in the outer region. It is clear that the third term on the right-hand side, which is given by the sum, is only relevant to the unsteady effects. Without the third term, the boundary condition (10) coincides with the steady IBC obtained in [20]:

$U^{\prime}=V_{0}^{\prime}+\bar{V}^{\prime} U$ 


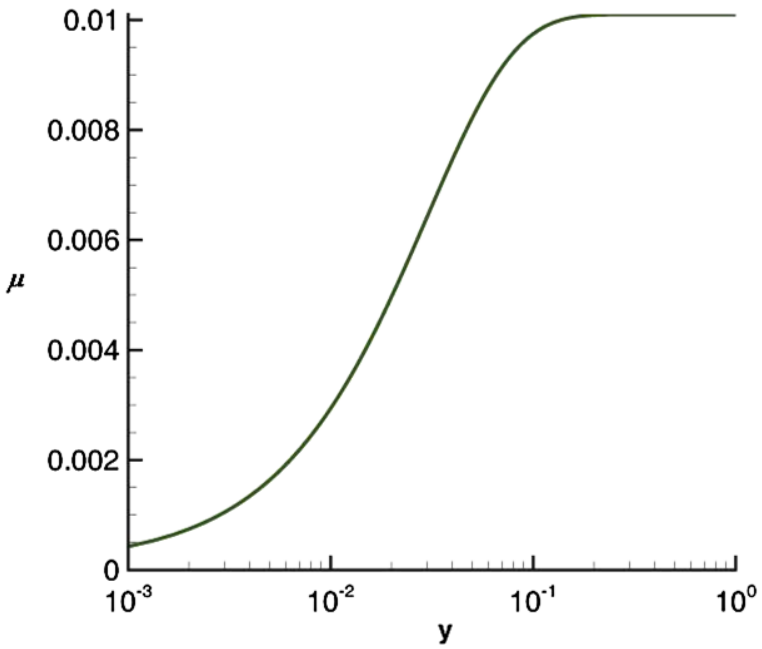

Fig. 1. Viscosity profile: $R e=100$.

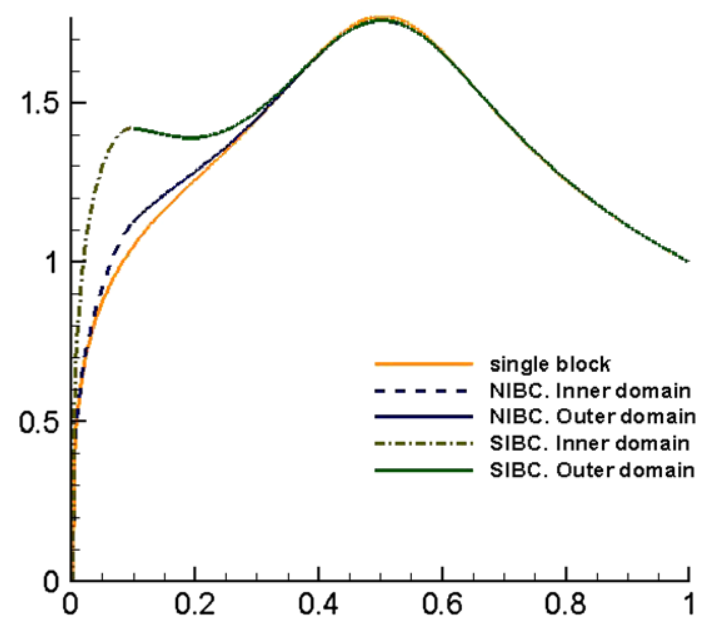

Fig. 2. Profile at $t=1$. Solid line is single block solution; dashed and solid line corresponds to $N=1$; dashed-dotted and solid line, $N=0$.

Thus, instead of the original IBVP in the domain $\Omega$, we can first solve the IBVP in the domain $\Omega^{+}$with the IBC set at $y=\delta$. Then, if required, we can obtain the solution in the domain $\Omega^{-}$represented by Eq. (9).

The entire algorithm consists of the following steps.

$1^{0}$. Solution of the spectral problem.

One possible algorithm to tackle this problem is as follows.

It starts from an initial approximation $u^{(0)}$ satisfying the homogeneous boundary conditions. At an $n$-th iteration

$\lambda^{(n)}=\frac{1}{2}\left(u^{(n) 2}, v^{\prime}\right)+\left(\mu u_{y}^{(n)}, u_{y}^{(n)}\right)$.

Then, the next prediction is obtained from the solution of equation

$L_{y} u^{(n+1)}=-\lambda^{(n)} u^{(n+1)}$

with homogeneous boundary conditions.

As soon as the iterative process converges, we obtain the first eigenfunction $\Psi^{(1)}$ and eigenvalue $\lambda^{(1)}$. Next, we consider the space orthogonal to the eigenfunction to find out the next eigenfunction. The algorithm is then repeated. Each time we consider the subspace orthogonal to the eigenfunctions have been obtained.

$2^{0}$ Next, we calculate the basis functions $V_{0}$ and $V$ from BVPs (5) and (6).

$3^{0}$. The IBC is calculated from (10).

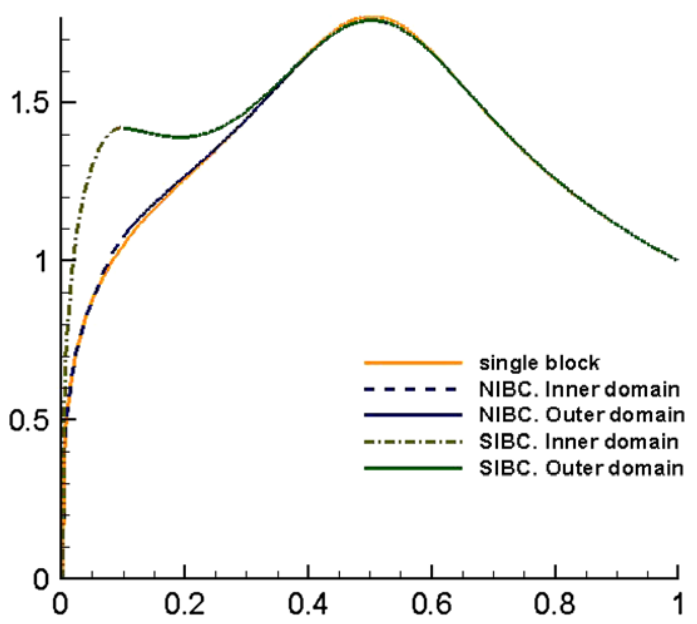

Fig. 3. Profile at $t=1$. Solid line is single block solution; dashed and solid line corresponds to $N=2$; dashed-dotted and solid line, $N=0$.

$4^{0}$. The BVP in the outer domain is solved.

$5^{0}$. The solution in the inner domain is obtained if required.

Now, let us consider the effect of unsteady terms in the IBC (10) for a number of test cases.

\section{Test cases}

The test cases were considered for the following input parameters for Eq. (1): $c_{0}=1, c_{1}=1, \beta=2, \operatorname{Re}=10^{2}, \epsilon=0.03$, $\epsilon_{0}=0.01$, while the interface boundary was set at $\delta=0.1$. The appropriate viscosity profile is shown in Fig. 1.

The boundary conditions introduced in (2) were as follows:

$U(0, t)=0, \quad U(1, t)=1$.

The initial profile was set to be linear:

$U(y, 0)=y$.

The boundary condition was transferred from the left-hand side boundary $y=0$ to the interface boundary $y=\delta$. The effect of nonstationary terms in the IBC (10) was analyzed via comparison against the steady IBC (11). In the case of unsteady IBC, different number of Fourier harmonics $N$ was considered.

In the next figures, the solutions are represented both in the inner and outer domains regarding the stationary IBC (SIBC) and nonstationary IBC (NIBC). In the first series of test cases the driving force is not taken into account.

In the next three figures, the profiles are shown for $t=1$. In Fig. 2, the solid line represents the single-block benchmark solution; the dashed-solid line corresponds to the composite solution with $N=1$. For the comparison, the composite solution with steady $\operatorname{IBC}(N=0)$ is given by the dashed-dotted-solid line. One can see a significant error in the case of the steady IBC while the unsteady IBC gives a reasonably good prediction. This prediction can be improved if we increase the number of Fourier harmonics to $N=2$ as shown in Fig. 3. Starting from $N=3$, the composite solution graphically coincides with the benchmark solution.

By $t=10$, the solution becomes stationary. As shown in Fig. 4 , the composite solution with the steady IBC coincides with the benchmark solution.

In the case of a highly oscillatory driving force the difference between the steady and unsteady IBCs is significantly more essential. Consider the following driving force

$f=\sigma(1-\exp (-\alpha y)) \sin (\omega y) \sin (\omega t)$

with $\sigma=2 \times 10^{3}, \omega=9.42 \times 10^{2}$. 


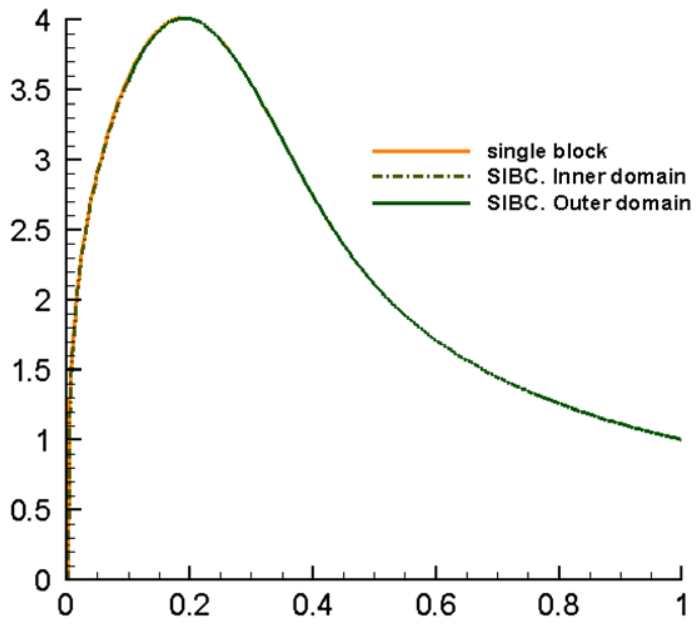

Fig. 4. Profile at $t=10$. Solid line is single block solution; dashed-dotted and solid line corresponds to the steady IBC.

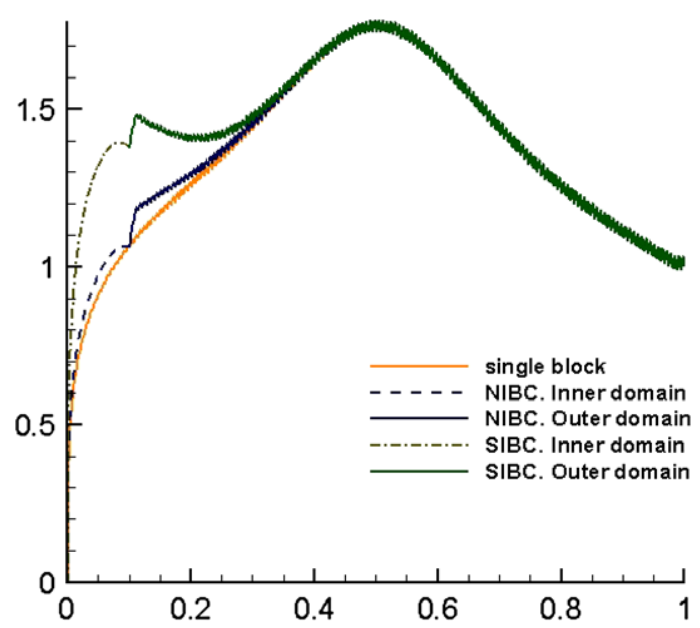

Fig. 5. Profile at $t=1$ with an oscillatory driving force. Solid line is single block solution; dashed-dotted and solid line corresponds to the steady IBC; dashed and solid line corresponds to $N=1$.

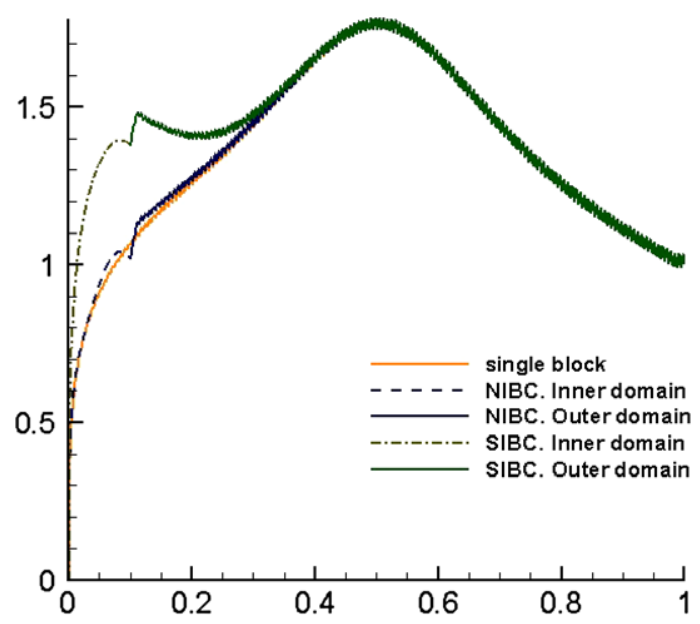

Fig. 6. Profile at $t=1$ with an oscillatory driving force. Solid line is single block solution; dashed-dotted and solid line corresponds to the steady IBC; dashed and solid line corresponds to $N=3$.

The driving force is not taken into account in the inner domain. This is similar to LES approach combined with RANS or wall functions [8]. The neglect of the driving force can make an essential

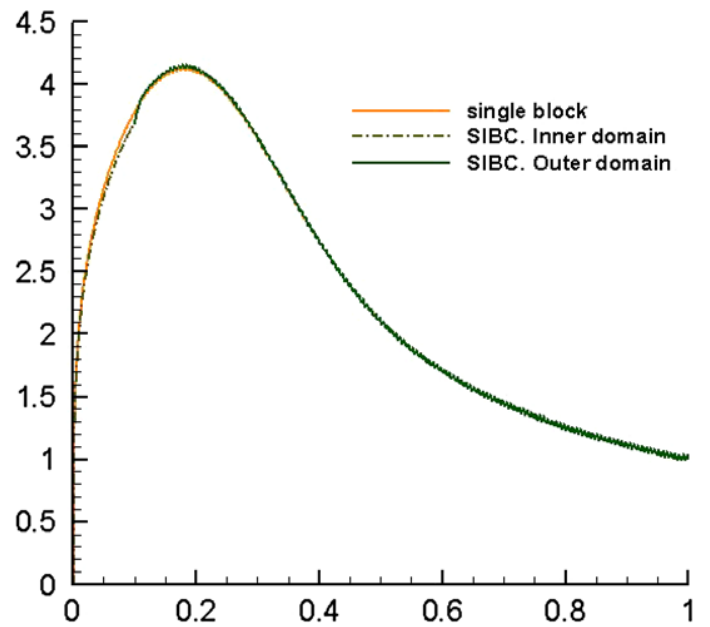

Fig. 7. Profile at $t=10$ with an oscillatory driving force. Solid line is single block solution; dashed-dotted and solid line corresponds to the steady IBC.

effect on the solution especially in the case of the steady IBC. In Fig. 5, the solid line corresponds to the single block solution. One can see that the solution significantly oscillates around the unperturbed solution. In the case of the steady IBC, the error is significantly more than that in the unperturbed case. The unsteady IBC performs much better even with $N=1$. However, the error is also more significant in comparison to the unperturbed case (see Fig. 2). The increase of $N$ to three allows us to improve the accuracy as shown in Fig. 6.

In all cases, there is a sharp drop of the solution nearby the interface boundary because the contribution of the driving force is not taken into account in the boundary conditions. The behavior of the solution reminds the well-known Gibbs effect or a ringing artifact in signal processing. At $t=10$, this effect is not essential as the mean flow is steady. As shown in Fig. 7, the steady and unsteady IBCs give the same solution.

Finally, it is worth noting that a similar effect occurs in the zonal approach RANS-LES approach where RANS does not spark turbulence in LES [11]. To overcome this problem, one can consider adding synthesized turbulent fluctuations [8] to the IBC for triggering the LES equations to resolve turbulence in the outer domain. However, this effect cannot be represented by the current linear model.

\section{Conclusions}

The technique of the boundary condition transfer has been extended to unsteady problems. According to this approach, a non-overlapping domain decomposition is realized. In this way, the inner domain with high gradients can be separated from the outer domain. At the interface boundary, the boundary conditions can be obtained as the result of the transfer of the boundary condition from the real boundary to an interface one. The boundary conditions depend on neither the solution in the outer domain nor the boundary conditions at the external boundary. Thus, the outer domain can be calculated for a different right-hand side and boundary conditions with the same IBC.

The test cases demonstrate that the transfer of the boundary condition that does not take into account unsteady effects can lead to a significant error. This result is very important in application to turbulence modeling based on the use of wall functions that are always derived in a stationary formulation.

In the future work the suggested approach will be applied to the URANS equations to simulate essentially unsteady flows. It seems the technique also has a potential to be used in LES to avoid timeconsuming near-wall computations. However, in the latter case 
they should be significantly elaborated yet to overcome the nonphysical effects that intrinsic to near-wall layer models.

\section{Acknowledgment}

The work has been partially supported by the Russian government under grant "On Measures to Attract Leading Scientists to Russian Educational Institutions" (contract No. 11.G34.31.0072).

\section{References}

[1] T. Kopp, Proceedings of ECCOMAS CFD'2006, The Netherlands, 2006

[2] S.V. Patankar, D.B. Spalding, Heat and Mass Transfer in Boundary Layers, Morgan-Grampian Press, London, 1967.

[3] H. Grotjans, F.R. Menter, in: K.D. Papailiou (Ed.), Proceedings of Computational Fluid Dynamics'98, ECCOMAS, 1(2), John Wiley \& Sons, Chichester, UK, 1998, pp. $1112-1117$.

[4] G. Kalitzin, G. Medic, G. Iaccarino, P.A. Durbin, J. Comput. Phys. 204 (1) (2005) 265-291.

[5] T.J. Craft, S.E. Gant, H. Iacovides, B.E. Launder, Numer. Heat Transfer 45 (2004) $301-318$

[6] S.V. Utyuzhnikov, Int. J. Comput. Fluids 38 (9) (2009) 1710-1717.
[7] AIAA J. 17 (1979) 1293-1313.

[8] L. Davidson, M. Bilson, Int. J. Heat and Fluid Flow 27 (2006) 1028-1042.

[9] M.L. Shur, Ph.R. Spalart, M.Kh. Strelets, A.K. Travin, Int. J. Heat and Fluid Flow 28 (2008) 1638-1649.

[10] D. Drikakis, B. Geurts (Eds.), Turbulent Flow Computation, Kluwer Academic Publishers, 2002, p. 369.

[11] P. Sagaut, S. Deck, M. Terracol, Multiscale and Multiresolution Approaches in Turbulence, Imperial College Press, 2006, p. 340

[12] U. Piomelli, Prog. Aerosp. Sci. 44 (2008) 437-446.

[13] W. Cabot, Annual Research Briefs 1995, Center for turbulence research, NASA/Stanford University, 1995: 4150.

[14] W. Cabot, P. Moin, J. Flow, Turbul. Combust. 63 (1999) 269-291.

[15] U. Schumann, J. Comput. Phys. 18 (1975) 376-404.

[16] U. Piomelli, J. Ferziger, P. Moin, J. Kim, Phys. Fluids 1 (1989) 1061-1068.

[17] D. Panara, M. Porta, T. Schoenfeld, European Conference on Computational Fluid Dynamics ECCOMAS CFD 2006, September 2006.

[18] P. Sagaut, Large Eddy Simulation for Incompressible Flows. In Introduction, third ed., Springer-Verlag, 1998, p. 556.

[19] C. Duprat, G. Balarac, O. Metais, P.M. Congedo, O. Bruglere, Phys. Fluids 23 (2011).

[20] S.V. Utyuzhnikov, Comput. \& Fluids 34 (7) (2005) 771-784.

[21] S.V. Utyuzhnikov, Int. J. Comput. Fluids 35 (10) (2006) 1193-1204.

[22] S.V. Utyuzhnikov, J. Appl. Numer. Math. 58 (10) (2008) 1521-1533.

[23] S.V. Utyuzhnikov, IMA J. Appl. Math. 74 (1) (2009) 128-148.

[24] V.S. Vladimirov, Equations of Mathematical Physics, Dekker, New York, 1971. 